\title{
A Novel Approach to Generate Up-sampled Tomographic Images using Combination of Rotated Hexagonal Lattices
}

\author{
Neha Dixit and Jayanthi Sivaswamy \\ International Institute of Information Technology,Hyderabad \\ Hyderabad,India - 500032. \\ Email: nehadixit@research.iiit.ac.in and jsivaswamy@iiit.ac.in
}

\begin{abstract}
Generation of upsampled tomographic images via combination of rotated lattices has been explored in [1]. In this paper, we evaluate the existing method using real phantom data. Up-sampled tomographic images are generated via combination of rotated hexagonal lattices. Sinogram data is filtered and back-projected on two hexagonal lattices which are rotated versions of each other. Samples from these lattices are interpolated to generate an up-sampled image defined on a square lattice. These results are compared with direct up-sampling method and image ISR-2 algorithm described in [10]. Two PET phantoms - NEMA and Hoffman brain phantom are used for purpose of evaluation. The results of the proposed method show considerable improvement over direct up-sampled image in terms of contrast, sharpness and imaging artifact; but when compared with ISR-2 generated image, the difference in image quality is not significant. A key advantage of the proposed method is that only two images are required for generating a high resolution image whereas ISR2 requires $k$ low resolution images for an up-sampling factor of $k$.
\end{abstract}

keywords: Super-resolution, Tomographic images, Combination of rotated lattices, Hexagonal lattice, Medical image processing.

\section{INTRODUCTION}

Functional imaging such as nuclear imaging (PET and SPECT) is used to estimate the functional aspects of organs, lesions, etc. During the imaging process, a limited amount of radionuclide is introduced into the patient. The strength of detected signal (projection or sinogram) is very weak which results in poor resolution (typically $128 \times 128$ ) and decrease in signal to noise ratio (SNR) of the reconstructed image [2]. In this paper, we concentrate mainly on PET imaging. The low resolution of PET images impede detection of finer details such as very small tumors [3],[4]. Additionally, fusing PET and MRI images, which are twice as bigger as PET images is challenging and up-sampling is needed to match the MRI image size prior to registration [5]. Preserving the details of the PET image after up-sampling is also vital for clinical and detection purpose. In general, linear interpolation method is used for up-sampling of PET image. Although this method is computationally inexpensive, it deteriorates the image quality.

An alternative method for enhancing quality of medical images is "union of shifted lattices" [6]. In this method, the image resolution is improved by a combining low resolution images with spatial shifts (translation), which provide additional information to improve quality and the contrast ratio of the clinical images. This method has been implemented in the past for improving resolution of both CT and PET images. For fan-beam CT, Izen et al. [7] have shown that the shifted lattices can be generated by standard fan-beam lattice and its reflected lattice. The union of these lattices have been used to increase the resolution by a factor of two. Kennedy et al. [8] have used low resolution PET images generated from projections acquired by shifting and rotating the detectors by subpixel. Chang et al. [9] proposed an improvement over [8] by generating low resolution images from one sinogram by back-projecting it onto shifted (by subpixel) lattices. Ideally, $k$ times upsampled image is generated by combining, $k^{2}$ subpixel shifted images. To decrease the compuational cost in [10] chang proposed two new algorithm ISR-1 (incomplete super resolution) and ISR-2 which generates high resolution images by combining $(2 k-1)$ and $k$ low resolution images without degrading the image quality. In general, these methods aim to combine non overlapping samples on a low resolution grid to create a higher resolution image.
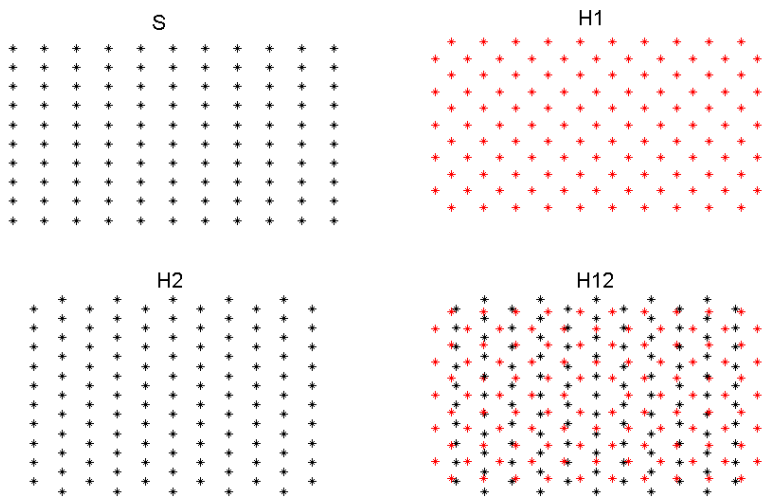

Fig. 1: Samples on square, hexagonal (top row); rotated hexagonal and combination of hexagonal lattices (bottom row).

In 2-D, however, non-overlapping samples can be generated by translation or rotation. While the above methods focus on translation there has not been any work reported on rotation. As explained in [1], we combine the information from two different lattices which are rotated versions of each other in order to generate an up-sampled image. We also examine the use of a hexagonal lattices, rather than the traditionally used square lattices, as a starting point. The choice in favor of hexagonal (over square) lattice is motivated by several factors - it offers superior packing efficiency, uniform connectivity and additional equi-distant neighbors. These factors have been 
shown to lead to better representation for curves among other benefits [13]. These factors have been exploited for image reconstruction. Reconstruction algorithms implemented using hexagonal lattice have been shown to yield images with enhanced quality [11] and to incur reduced computational cost [12], since hexagonal lattice requires 13 percent less samples than square lattice to represent an image. More recently, Faille and Petrou [14] have used hexagonal grid to reconstruct image from very sparse set of non-uniform samples via linear splinebased interpolation. The result of this reconstruction is of good image quality in spite of the sparsity of the samples.

\section{METHOD}

Consider samples defined on two lattices which are rotated versions of each other. Let $A$ and $A_{\theta}$ be the sampling matrices of the lattices, which are related by

$$
A_{\theta}=T_{\theta} \times A
$$

where $T_{\theta}$ is the rotation (by $\theta$ ) matrix. Fig. 1 shows the samples generated for different instances of $\mathrm{A}$. Here, $\mathrm{S}$ represents the case when $\mathrm{A}$ is a square lattice while $\mathrm{H} 1$ and $\mathrm{H} 2$ show the samples generated by a hexagonal and rotated (by $\theta=30^{\circ}$ ) hexagonal versions of A. Finally, H12 shows the samples of $\mathrm{H} 1$ overlaid on $\mathrm{H} 2$. It can be observed from $\mathrm{H} 12$ that the samples are no longer regularly spaced but are quasi-periodic. Our strategy is to use this set of samples to generate samples on a higher resolution square grid. Following the findings in [14], we can expect to have a good quality reconstructed image.

In the present context, we propose to derive the samples on $\mathrm{H} 1$ and $\mathrm{H} 2$ from sinograms via filtered back-projection (FBP), a standard procedure used in tomographic reconstruction. This is described in the next section. Since signal acquisition in PET is a stochastic process, PET image reconstruction traditionally is implemented using iterative reconstruction methods like maximum likelihood -expectation maximization (ML-EM) or ordered subsets expectation maximization (OSEM). These methods require an initial estimate of reconstructed image to begin with, for which image generated using FBP is considered as the best possible option [16].

\section{A. Filtered back projection}

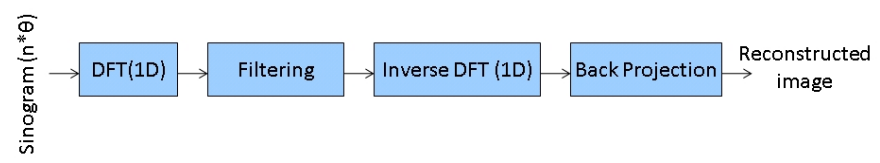

Fig. 2: Processing stages in filtered back projection

Reconstruction of an image from sinogram data of size $n \times \theta$, (n is the number of detectors and $\theta$ is the number of angular samples) requires two steps, first, back projecting the sinogram into a continuous image and then sampling it to generate a discrete image [9]. The filtered back projection algorithm consists of four steps as shown in Fig.2. It is possible to generate the samples on a lattice at arbitrary orientation $\theta$ using this algorithm. We utilize this fact to generate the desired samples on $\mathrm{H} 1$ and $\mathrm{H} 2$ which constitute two images.

\section{B. Pipeline-1 for generating upsampled image}

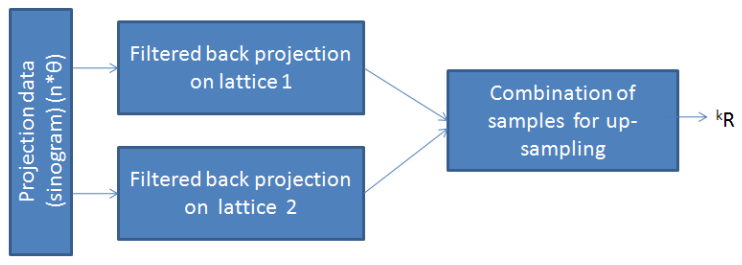

Fig. 3: Architecture of pipeline-1.

The two reconstructed images $\mathrm{H} 1$ and $\mathrm{H} 2$ are combined using a suitable interpolation method to generate an upsampled image ${ }^{k} R$, where $k$ is sampling factor. The complete pipeline of processing for generating upsampled image is shown in Fig.3.

\section{Validation of results}

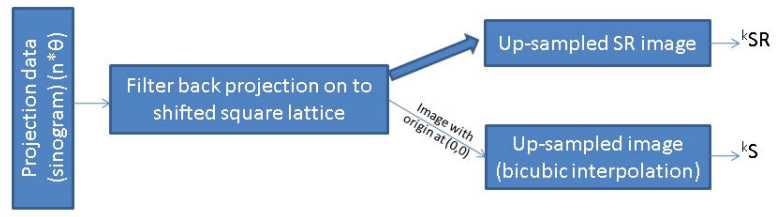

Fig. 4: Architecture of pipeline-2.

The second pipeline was constructed to validate the results of the proposed method. Here two up-sampled images were generated using direct up-sampling of the reconstructed image $\mathrm{S}$ and super resolution algorithm ISR-2 [10]. Fig.4 shows this second pipeline.

a) Direct method: The FBP algorithm was applied to the input sinogram, which was filtered and back projected onto a square lattice. This was next up-sampled using bi-cubic interpolation to obtain image ${ }^{k} S$.

b) Super resolution (SR) method: The FBP algorithm was used to generate sub-pixel shifted images defined on square lattices. The number of such generated images depends on the up-sampling factor $k$ and the SR algorithm. For example, to up-sample by a factor of 4 via a basic SR method (based on union of shifted lattices) 16 images are required. However, ISR1 and ISR2 algorithms described in [9] require only 7 and 4 images respectively. These images are used to generate up-sampled image ${ }^{k} S R$ via SR technique.

\section{Implementation}

The pipeline-1 was implemented as follows- angular shift between the images was fixed to $30^{\circ}$, to ensure minimum overlap between the samples. For best possible distribution of samples, hexagonal lattices with basis vectors $(1,0)(1 / \sqrt{3}, 1)$ and $(1,1 / \sqrt{3}),(0,1)$ were used. All the final images were 
generated on a square lattice. For generating up-smapled $\mathrm{R}$ image from $\mathrm{H} 12$ non-uniform thin plate spline interpolation kernel was used. In pipeline-2, sub-pixel shifted images were reconstructed using Fessler's toolbox [16]. While generating ${ }^{k} S R$ image, for $\mathrm{k}=2$, four shifted images were combined with origin at $(0,0),(0.5,0),(0.5,0)$ and $(0.5,0.5)$; but for $k=4$, the approach in ISR-2 was incorporated. Thus four (instead of sixteen) images with center at $(0,0),(0.25,0.25),(0.5,0.5)$ and $(0.75,0.75)$ were generated. Samples from these low resolution images were interpolated using a bicubic kernel to generate desired samples on a high resolution grid (upsampled image) using Vanderwalle's toolbox [15].

\section{RESULTS}

Two PET phantoms were selected for our study- NEMA and Hoffman brain phantom. NEMA sinogram is of size $293 \times 280$ , whereas Hoffman brain phantom is a 3-D phantom of size $367 \times 315 \times 91$, where 91 is the number of slices. The results of the experiments were compared quantitatively using line (tangential/radial) profiles, noise reduction and energy distribution plot. Line profiles help to compare the contrast difference between the reconstructed images. Energy distribution plot were generated using IQM toolbox [17], computed from the normalized power spectrum of an image shows the energy distribution in the radial direction ( $\rho=64$ bands). To suppress the noise content sinograms were filtered with one dimensional Gaussian kernel of size 3 designed using [16].

1) NEMA phantom: NEMA phantom was used to study the contrast of the up-sampled image and the effectiveness of an up-sampling method in suppressing imaging. Fig.5 (a), (c) and (e) show the up-sampled ${ }^{4} S,{ }^{4} R$ and ${ }^{4} S R$ images (up-sampling factor 4) of the NEMA phantom while (b), (d) and (f) show their corresponding amplitude spectra. While upsampling an image, our aim is to preserve details and definition of the image and prevent imaging. The energy distribution plot shown in Fig.5, illustrates a smooth variation for the image ${ }^{4} R$ obtained by our proposed method. In contrast, the energy distribution of the images ${ }^{4} S$ and ${ }^{4} S R$ have prominent irregularities beyond the baseband, thus indicating presence of imaging. Fig.6, (a) and (b) illustrate the intensity variation in the radial and tangential directions across the smallest circle (sphere) in the phantom. A quantitative evaluation of

TABLE I: Contrast ratio across the smallest sphere

\begin{tabular}{|l|l|l|}
\hline Images & Radial & Tangential \\
\hline$S^{4}$ & 0.7263 & 0.6952 \\
$R^{4}$ & 0.7649 & 0.7143 \\
$S R^{4}$ & 0.7354 & 0.7037 \\
\hline
\end{tabular}

the contrast was carried out by taking the intensity profile of the smallest sphere of the image and using the contrast ratio [10] defined as follows.

$$
C R=\frac{C-T}{C}
$$

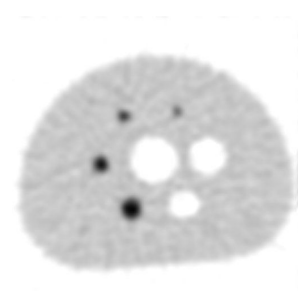

(a) ${ }^{4} S$

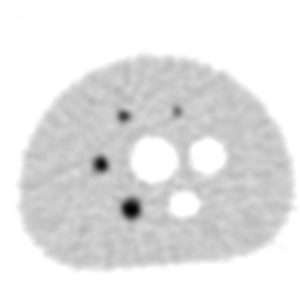

(c) ${ }^{4} R$

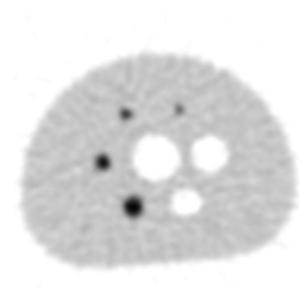

(e) ${ }^{4} S R$

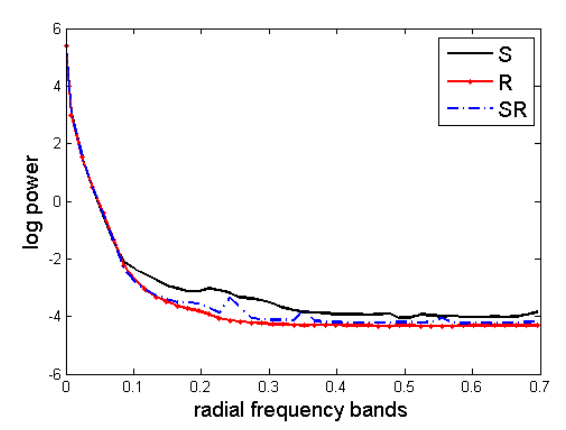

(g) energy plot

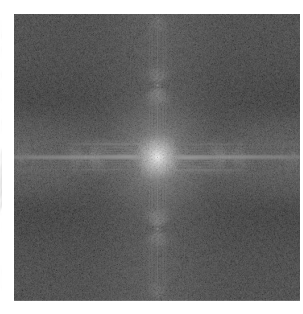

(b) ${ }^{4} S$

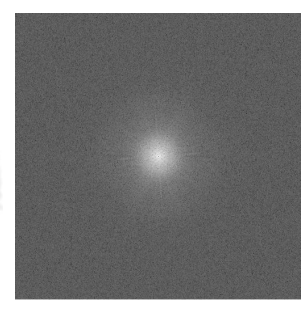

(d) ${ }^{4} R$

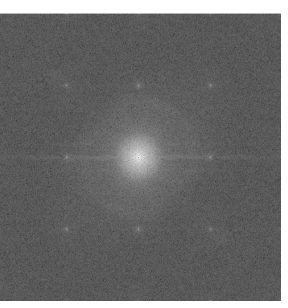

(f) ${ }^{4} S R$
Fig. 5: Upsampled results for NEMA phantom and their corresponding amplitude spectra (a) through (f).(g) Energy plot of ${ }^{4} S,{ }^{4} R$ and ${ }^{4} S R$

$C$ is the amplitude of the crest and $T$ is the amplitude value of the trough. The obtained contrast values are tabulated in Table.I. From this table it is seen that $\mathrm{R}$ increases the contrast of the reconstructed images.

2) Hoffman brain phantom: The Hoffman phantom was used mainly to study the effect of up-sampling on the quality of image in terms of noise content and contrast. Out of 91 slices, 2 slices, namely the 19th and 24th, are reconstructed. Fig.7 show the up-sampled (by 4) images of the 24th slice of the Hoffman phantom and their corresponding amplitude spectra. It can be observed from these figures that the energy 


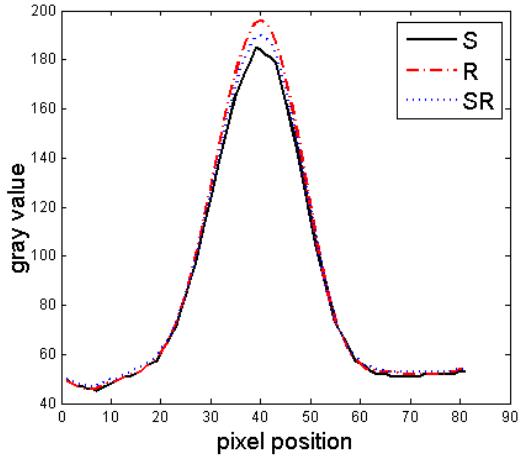

(a) radial

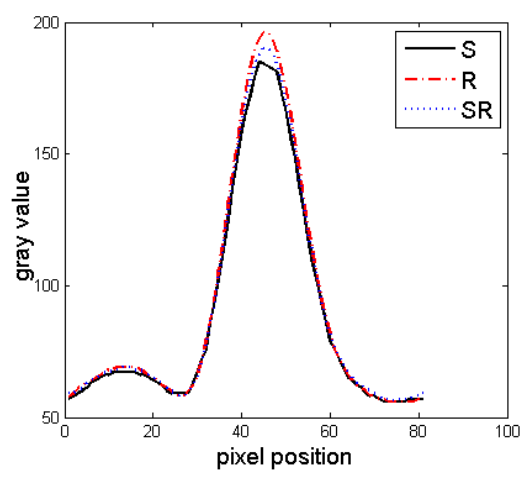

(b) tangential

Fig. 6: (a) Radial and (b) tangential line profiles of the smallest sphere in the NEMA phantom

distribution plots for the Hoffman and NEMA phantoms are similar. The distribution for $\mathrm{R}$ is smoother as compared to $\mathrm{S}$ and $\mathrm{SR}$, which means that $\mathrm{R}$ is able to suppress imaging when compared with $\mathrm{S}$ and $\mathrm{SR}$. In contrast, the distribution for $\mathrm{S}$ and SR shows prominent variations which signifies presence of imaging.

Fig. 8 (b) shows a line profile in the upsampled images, for a selected line in Fig. 8 (a). To enable noting the difference in upsampled images, Table I shows the gray value difference (SR-S and R-S), in selected regions of these profiles: a trough region and a crest region. This should be positive for crest region and negative for trough region. Improvement in contrast (over S) in SR and $\mathrm{R}$ images can be noted clearly from the average difference shown in this table. Since upsampling by combining low resolution images can introduce noise, we studied a noisy ROI. Fig. 9 (a) shows a ROI of size $41 \times 41$ reconstructed from original sinogram (with no smoothing) of the 19th slice. The DC suppressed noise spectra for ${ }^{2} S^{2} R$ and ${ }^{2} S R$ images in Fig 9 (b) to (d) show that the noise content in $\mathrm{SR}$ and $\mathrm{R}$ is circularly symmetric and in comparable to $\mathrm{S}$.

\section{DISCUSSION AND CONCLUSION}

In this paper, we have used real phantom for evaluating the said method [1] which could be used to generate upsampled tomographic images by combining samples from two rotated hexagonal lattices. The results of the proposed method were compared against ISR-2 algorithm [10] and

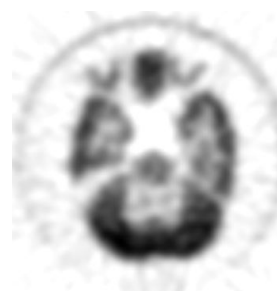

(a) ${ }^{4} S$

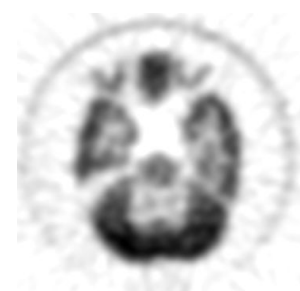

(c) ${ }^{4} R$

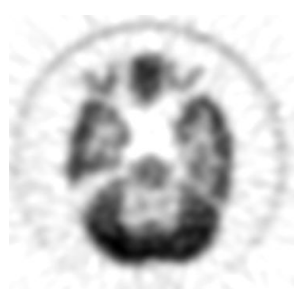

(e) ${ }^{4} S R$

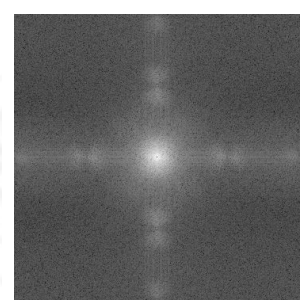

(b) ${ }^{4} S$

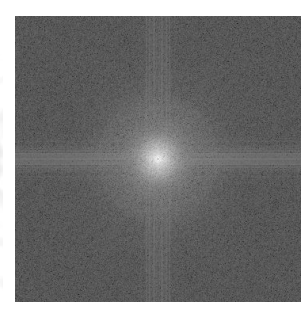

(d) ${ }^{4} R$

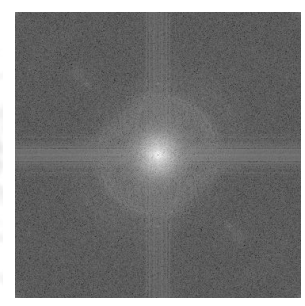

(f) ${ }^{4} S R$

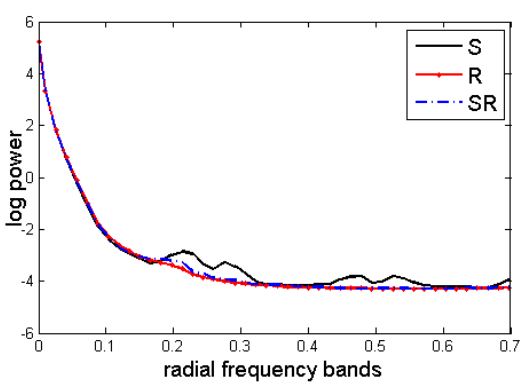

(g) energy plot

Fig. 7: Upsampled results of 24th slice of Hoffman phantom and their corresponding amplitude spectras. (g) Energy plot of ${ }^{4} S,{ }^{4} R$ and ${ }^{4} S R$

direct up-sampling. Results show that the proposed scheme produced image of better quality when compared with direct up-sampling method as well as of similar quality as obtained with ISR-2 algorithm.

In [10], Chang has discussed the disadvantages of using union of shifted lattices (USL) for generating super resolution images in terms of computational cost and storage requirements. For up-sampling an image by factor $k$, USLbased method requires $k^{2}$ images, where as ISR-1 and ISR2 algorithms (proposed by Chang) require $2 \times k-1$ and $k$ images respectively without affecting the quality of upsampled images. Our proposed method offers further reduction as only two images for any up-sampling $(\mathrm{k}=2,3,4 \ldots)$ factor. This amounts to considerable savings in computational cost. 


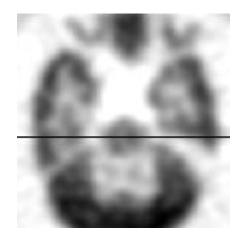

(a) selected line

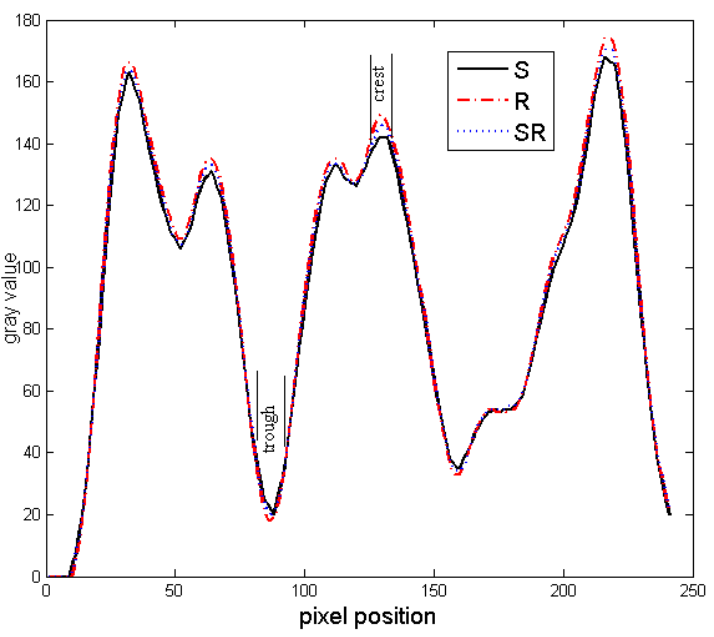

(b) line profile

Fig. 8: Line profile of ${ }^{4} S,{ }^{4} R$ and ${ }^{4} S R$ of 24th slice of Hoffman phantom

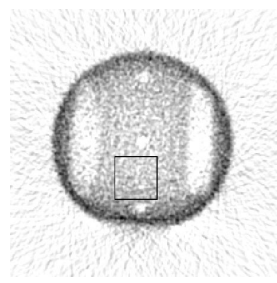

(a) ROI

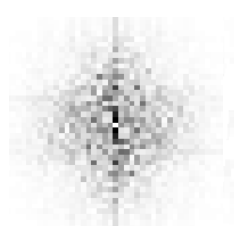

(b) ${ }^{2} S$

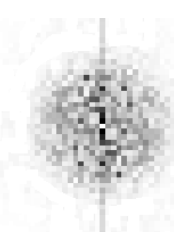

(c) ${ }^{2} R$

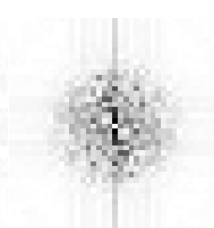

(d) ${ }^{2} S R$
Fig. 9: (a) Shows ROI of 19th slice of Hoffman phantom used for computing noise spectra, (b),(c)(d) Noise spectra of the ROI

For instance, for $k=4$, this reduction is at least $50 \%$. There is also a reduction in minimum required storage space when compared to existing methods.

Visually, the up-sampled images S, SR and R for both NEMA and Hoffman phantoms appear to be the same. However, line profiles indicate contrast improvement. From the line profiles of of both the phantoms it can also be concluded that the contrast of $\mathrm{R}$ and $\mathrm{SR}$ is better than $\mathrm{S}$. It is evident from the noise spectra of Hoffman phantom that rotated lattices and union of shifted lattices reduce the background noise relative to $\mathrm{S}$.

In general, the main objective of generating up-sampled images by combination of low resolution images is to improve the quality and suppress imaging artifacts. Based on our results on NEMA and Hoffman phantom images it can be concluded that up-sampling from the space of combination of rotated lattices produces results of comparable quality to SR techniques however with lower computational requirement.

TABLE II: Difference in gray values of marked region 1 and 2 in $8(b)$

\begin{tabular}{|c|rrrrrrrrrrr|r|}
\hline Images & \multicolumn{10}{|c|}{ Difference in gray values of trough region } & Avg \\
\hline R-S & -2 & -2 & -3 & -2 & -4 & -5 & -3 & -1 & -2 & -3 & -1 & -2.5 \\
SR-S & -1 & -1 & -2 & 0 & -2 & -2 & -1 & +1 & 0 & -2 & -1 & -1.0 \\
\hline Images & \multicolumn{11}{|c}{ Difference in gray values of crest region } \\
\hline R-S & +5 & +7 & +7 & +7 & +7 & +6 & +4 & +5 & +5 & +5 & +5 & 5.7 \\
SR-S & +2 & +3 & +3 & +3 & +4 & +4 & +3 & +3 & +3 & +3 & +2 & 3.0 \\
\hline
\end{tabular}

\section{REFERENCES}

[1] N.Dixit, N.V.K. Medathati, J.Sivaswamy, "Synthetic Zooming of Tomographic Images by Combination of Lattices", Accepted at Nuclear Science Symposium Medical Imaging Conference, IEEE, 2009.

[2] Jiri and Jan, "Medical image processing, reconstruction, and restoration, concepts and methods", CRC Press, 2006.

[3] Y.Nie, Q.Li, F.Li, Y.Pu, D.Appelbaum, and K. Doi, ”Integrating PET and CT information to improve diagnostic accuracy for lung nodules: A semiautomatic computer-aided method,"J. Nucl. Med.,vol. 47 ,2006, pp 10751080

[4] S.K.Kim, M.Allen-Auerbach, J.Goldin, B.J.Fueger, M.Dahlbom, M.Brown, J.Czernin, and C.Schiepers, Accuracy of PET/CT in characterization of solitary pulmonary lesions,J. Nucl. Med. vol. 48,2007, pp 214220

[5] R.Mayers."The application of PETMR image registration in the brain", The British Journal of Radiology, vol. 75, pp S31-S35, Special Issue 2002

[6] H.Behmard, "Reconstruction of 2-D signals from union of shifted lattices", Proc. ICASSP,vol. 4, 2005, pp 197-200.

[7] S.H.Izen, D.P.Rohler and K.L.A. Sastry, "Exploiting symmetry in fan beam CT: Overcoming third generation undersampling" SIAM J. Appl Math, vol.65, 2005, pp 1027-1052.

[8] J.Kennedy, O.Israel, A.Frenkel, R.Bar-Shalom, and H.Azhari, "Superresolution in PET imaging",IEEE Trans Medical Imaging vol. 25, 2006, pp 137-147.

[9] G.Chang, T.Pan, J.Clark, Jr, OR Mawlawi, "Comparison between two super-resolution implementations in PET imaging", Med. Phys vol. 36, 2009, pp 1370-1383.

[10] G.Chang, T.Pan, J.Clark, Jr, OR Mawlawi, "Optimization of superresolution processing using incomplete image sets in PET imaging" , Med. Phys vol. 35, 2008, pp 5748-5757.

[11] A.Yabushita,K.Ogawa. "Image reconstruction with hexagonal grid" Nuclear science symposium, vol. 3, 2002, pp 1500-1503.

[12] M.Knaup, S.Steckmann, O.Bockenbach, M. Kachelrie ,'CT image reconstruction using hexagonal grid", Nuclear science symposium, Vol 4, 2007, pp 3074-3076.

[13] L.Middleton, J.Sivaswamy, "Hexagonal Image Processing: A Practical Approach", New York: Springer, 2005.

[14] F.Faille and M.Petrou, "Bio-inspired,Invariant Image Reconstruction from Irregularly Placed Samples", British Machine Vision Conference, 2008.

[15] P.Vanderwalle, K.Krichane, P.Zbinden, Super resolution toolbox (http://lcavwww.epfl.ch/software)

[16] J.A.Fessler, image reconstruction toolbox (http://www.eecs.umich.edu/ fessler/code)

[17] N.B. Nill and B.H. Bouzas, "Objective Image Quality Measure Derived from Digital Image Power Spectra", Optical Engineering, vol.31, pp.813825, April 1992. 